\title{
Neuroprotection: VEGF, IL-6, and clusterin: the dark side of the moon
}

\author{
S. Pucci ${ }^{1, *, a}$, P. Mazzarelli ${ }^{1}$, F. Missiroli ${ }^{2}$, F. Regine ${ }^{2}$ and F. Ricci ${ }^{2, * *, a}$ \\ ${ }^{1}$ Department of Biopathology, Institute of Anatomic Pathology, University of Rome "Tor Vergata", 00133 Rome, Italy \\ ${ }^{2}$ Section of Ophthalmology, Department of Biopathology, University of Rome "Tor Vergata", 00133 Rome, Italy
}

\begin{abstract}
Growth factors and their respective receptors are key regulators in development and homeostasis of the nervous system, and changes in the function, expression, or downstream signaling of growth factors are involved in many neuropathological disorders.

Recently, research has yielded a rich harvest of information about molecules and gene, and currently the assumption "a gene-a protein", where each gene encodes the structure of a single protein, is becoming a paradox. In the past years, the discovery of synergic or antagonistic proteins deriving from the same gene is a novelty upsetting. In some way, the conventional function of proteins involved in DNA repair, cell death/ growth induction, vascularization, and metabolism is inhibited or shifted toward other pathways by soluble mediators that orchestrate such change depending on the microenvironment conditions. In this chapter, we focus on the antithetic properties that proteins could exert, depending on the microenvironment that orchestrates the complex networks among proteins and their respective partners.
\end{abstract}

Keywords: VEGF; interleukin-6; clusterin; glaucoma

\section{Neuroprotection: VEGF-A, a shared growth factor}

Recently, the characterization of the molecular interactions among soluble factors in the microenvironment points out the action of some neuronal growth factors on the neovascular system (i.e., NGF, nerve growth factor; BDNF, brainderived neurotrophic factor). In the same way,

\footnotetext{
*Corresponding author. Tel.: + 39-06-20903953; Fax: +39-06-20902209; E-mail: sabinapuc@yahoo.it *Co-Corresponding author. Tel.: + 39-06-23188334; Fax: + 39-06-20902209;

E-mail: federico.ricci@uniroma2.it
}

\footnotetext{
${ }^{\mathrm{a}}$ These authors contributed equally to this work.
}

some vascular endothelial growth factors (VEGFs) first identified as endothelial specific mitogens exert a specific action on neuronal cells. The vascular and nervous systems are functionally and structurally different but they share some multiple similarities in the development, construction, and function. During development, the gradient of growth factors leads the long-distance final target. This chapter aims to point out the growing field of common growth factors, receptors, and inflammatory mediators within different networks that cooperate in the neuronal survival in stress conditions. The knowledge of the molecular interactions of these shared growth factors, pleiotropic but specific for the vascular and neural cells, is relevant for therapies addressed to the vascular and neurological diseases. 
Development and homeostasis of vascular and neuronal systems were believed to be controlled by different, specialized growth factors and their receptors that, in some cases, are the key regulatory steps by their own. Actually, different studies have shed light on the shared overlapping repertoire of growth factors that affect the development and homeostasis of the nervous and vascular systems. This suggests the cooperative evolution of molecular mechanisms to control path finding, spatial patterning proliferation, and survival. These include members of VEGF, ephrin, and neutrophin growth factor families. One prominent member among these is vascular endothelial growth factor-A (VEGF-A), an important multifunctional molecule with several important biological activities that depend on both the stage of development and physiological function of the organ in which it is expressed. The VEGF family comprehends six different homologous factors: VEGF-A-E and placenta growth factor (PIGF). All the components of this family are involved in the development of the vasculature and display multiple functions on endothelial cells; in particular, VEGF-A-C exert their specific action on neuronal cells. VEGF-A was first identified as a homodimeric protein of $32-42 \mathrm{kDa}$ that increased vascular permeability in the skin. The observation that tumor growth can be accompanied by increased vascularity was reported more than a century ago (see Ferrara, 2002). It was identified in the supernatant of a guinea pig tumor cell line and proposed as specific mediator of the hyperpermeability of tumor blood vessels, involved also in the formation of tumor-associated ascites. This proteinenhancing vascular permeability was named vascular permeability factor (VPF) (Senger et al., 1983). Further characterization of the bioactivity of this growth factor reveals that it exerts mitogenic effects also on vascular endothelial cells isolated from several districts. Due to its apparent target cell selectivity, this factor was renamed vascular endothelial growth factor (VEGF). By the end of 1989, Ferrara and coworkers reported the isolation of cDNA clones for bovine VEGF164 and three human VEGF isoforms: VEGF121, VEGF165, and VEGF189
(Leung et al., 1989). Additional reports showed the high conservation of VEGF across species, with approximately $85 \%$ homology between human and rat VEGF underlying the crucial role of this factor in the bioevolution (Conn et al., 1990).

\section{$V E G F-A$ isoforms}

The human VEGF-A gene is organized in eight exons, separated by seven introns, and is localized in chromosome 6p21.3 (Vincenti et al., 1996). Alternative splicing of the human VEGF-A gene gives rise to at least six different transcripts, encoding isoforms of 121-, 145-, 165-, 183-, 189-, and 206-amino-acid residues (Fig. 1; Tischer et al., 1991). Multiple protein forms are encoded through alternative exon splicing. All transcripts contain exons 1-5, which codify for the signal sequence and core VEGF binding or VEGF/PDGF homology domain, and exon 8 , with diversity generated through the alternative splicing of exons 6 and 7 . Exon 6 encodes a heparin-binding domain, whereas exons 7 and 8 encode a domain that mediates binding to neuropilin-1 (NP1) and heparin exons (Senger et al., 1983; Leung et al., 1989). Several additional minor splice variants also have been described including VEGF145 (Poltorak et al., 1997), VEGF162 (Lange et al., 2003), and VEGF165b, a variant reported to have an antagonistic effect on VEGF165a-induced mitogenesis (Bates et al., 2002). Recently identified VEGF165b displays different activities in respect to its isoform VEGF165a; it is not mitogenic and does not increase proliferation, but its functions are still not well characterized. Human VEGFA165, the most abundant and biologically active form, and VEGF-A121 are secreted as covalently linked homodimeric proteins, whereas the larger isoforms VEGF-A189 and VEGF-A206 are not readily diffusible and may remain sequestered in the extracellular matrix. Similar to VEGF165, VEGF121 that lacks heparin-binding properties is a freely diffusible protein (Plouet et al., 1989). VEGF189 and VEGF206 bind to heparin with particularly high affinity due to the presence of a highly basic 24-amino-acid insertion, and they are almost completely sequestered in the extracellular 
VEGF GENE ALTERNATIVE SPLICING

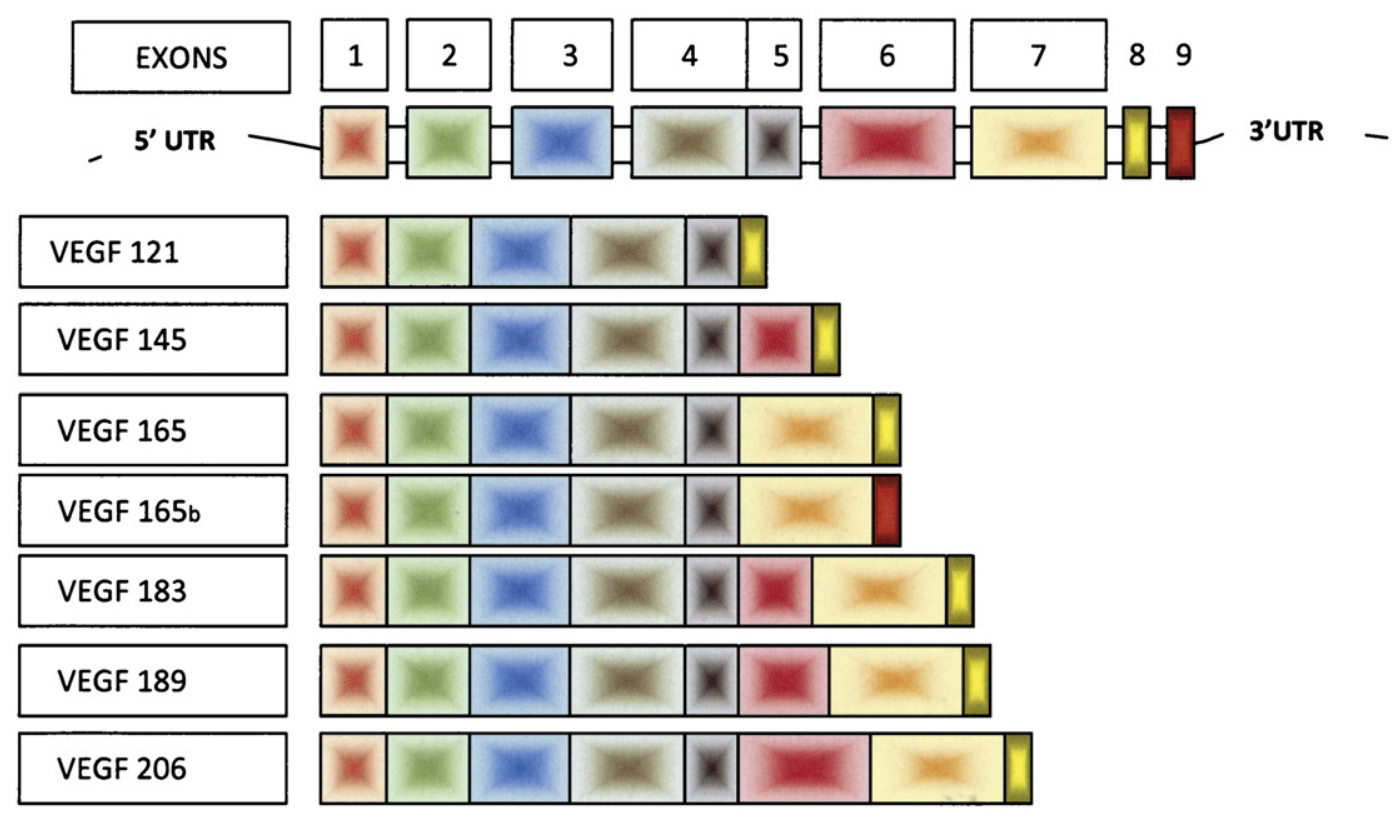

Fig. 1. VEGF-A isoforms. There are six different isoforms of VEGF-A arising by alternative exon splicing. VEGF165 induces and VEGF165b inhibits angiogenesis. All isoforms contain exons 1-5.

matrix (Houck et al., 1992; Park et al., 1993). Not only alternative RNA splicing but also extracellular proteolysis regulates the activity of VEGF. Proteolytic mechanisms are known to be important during pathologic angiogenesis, suggesting that non-heparin-binding cleaved forms of VEGFA may play an especially significant role (Pepper, 2001). The extracellular matrix-bound isoforms may be released in a diffusible form by plasmin cleavage at the $\mathrm{C}$ terminus, which generates a bioactive fragment (VEGF110), consisting of the first $110 \mathrm{~N}$-terminal amino acids. VEGF165 may also undergo a similar processing by plasmin (Houck et al., 1992). The C-terminal domain (111-165) of VEGF is critical for its mitogenic potency. More recent studies have shown that various matrix metalloproteases (MMPs), especially MMP-3, can also cleave VEGF165 to generate diffusible, non-heparin-binding fragments. Proteolytic processing of VEGF165 by MMP-3 takes place in sequential steps, with initial cleavage occurring at residues VEGF135, VEGF120, and, finally, VEGF113 (Lee et al.,
2005). Thus, the final product of MMP-3 processing, VEGF113, is expected to be biologically and biochemically very similar to the VEGF110, the plasmin-generated fragment. According to recent studies, levels of plasminogen and MMP-3 in the vitreous of patients affected by proliferative retinopathies are higher than those in controls, providing evidence for a prodegradative environment that may locally generate non-heparinbinding VEGF fragments.

\section{VEGF-A receptors}

The transduction pathway of VEGF-A is exploited by the binding of VEGF to its receptors. VEGF-A isoforms VEGF121, VEGF165a and VEGF165b, VEGF189, and VEGF206 are the products of alternative splicing where VEGF165 is the prevalent one. The differential splicing is influenced by the external growth factors and environmental conditions that guide the production of the predominant form needed in a particular district. The molecular factors that could influence this 
posttranscriptional modification that generates preferentially a specific isoform still need to be investigated. Every VEGF isoform displays a particular affinity for a class of receptors, which in turn activate preferentially a cascade of transduction pathways that lead to proliferative impulse, anti-cell death signals, and survival. The biological functions of VEGF are mediated via tyrosine kinase receptors, VEGF receptor-1
(Ftl-1), and VEGF receptor-2 (KDR/Flk-1). Additionally, certain VEGF isoforms bind to NPs, non-tyrosine transmembrane receptors (Fig. 2A).

In particular, NP1 is a non-tyrosine kinase receptor for VEGF165, the heparin-binding PIGF-2 isoform, VEGF-B, and VEGF-E. NP1 was first identified as a receptor for semaphorin-3A, a member of a family of polypeptides involved in axonal guidance and patterning; moreover, it is
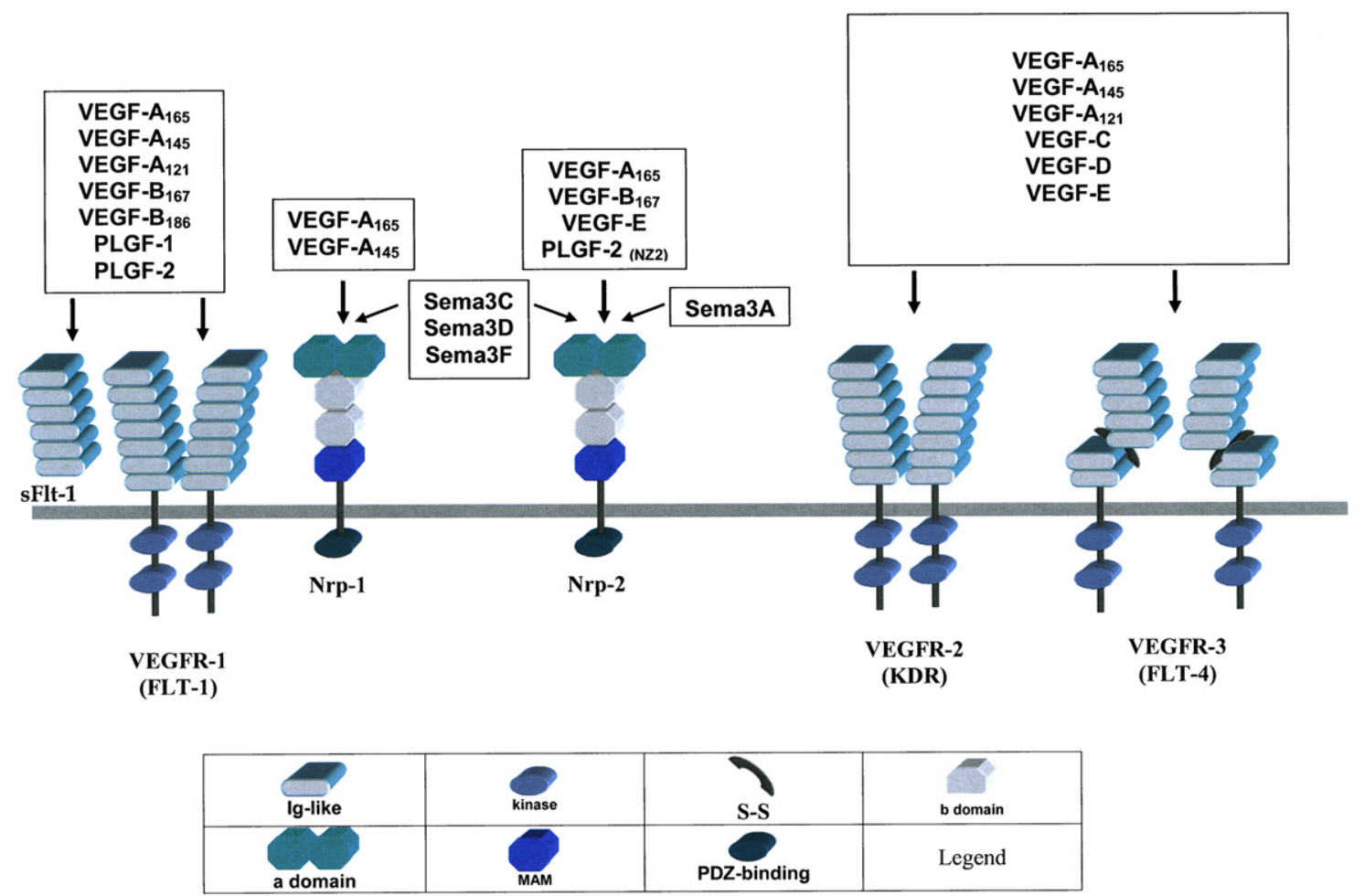

Fig. 2. (A) VEGF ligands and their receptors. VEGFR-1 (Flt-1) binds VEGF-A165, VEGF-A145, VEGF-A121, VEGF-B186, VEGFB167, PLGF-1, and PLGF-2 (human isoforms). The extracellular domain of VEGFR-1 is independently expressed as a soluble protein (sFlt-1 or sVEGFR-1), with predicted ligand specificity identical to that of the complete receptor. VEGFR-2 (KDR) is a receptor for VEGF-A165, VEGF-A145, VEGF-A121, the processed forms of VEGF-C and VEGF-D, and the viral VEGF-Es. Unprocessed VEGF$\mathrm{C}$ and VEGF-D bind VEGFR-3 (Flt-4) with higher affinity. NP1, a non-tyrosine kinase receptor for semaphorin 3A (Sema-3A), is also a co-receptor for VEGF-A165, PLGF-2, VEGF-B167, and VEGF-E(NZ2). NP2 binds only VEGF-A165 and VEGF-A145. (B) Neuronal receptors for VEGF and downstream signaling mechanisms. The major receptors for VEGF in neuronal cells are NP1 and VEGFR-2/ KDR. VEGF-A165 also competes with Sema-3A for binding to NP1. (Left) Associations between NP1 and the transmembrane proteins, plexin A1/2 and L1, are required for Sema-3A-induced growth cone collapse. L1 binds to both actin and ankyrin, which associates with spectrin and the associated actin cytoskeleton. Recruitment of the small Rho-like GTPase, Rnd, by the plexin A1 intracellular domain is sufficient to induce growth cone collapse and this is antagonized by RhoD. NP1 also associates with the PDZ domain protein, synectin/ NIP-1/GPIC. VEGF competition with Sema-3A may regulate associations between NP1 and either plexin A1/2 or L1, but this remains to be determined. Plexins have a large extracellular domain containing short cysteine-rich motifs; the plexin intracellular domain contains two separate regions of homology highly conserved within plexins but sharing little homology with other proteins. (Right) It is unknown whether VEGF binding to NP1 is sufficient to trigger intracellular signaling independently of Sema-3A. VEGF would stimulate activation of PI3K/Akt, PLC- $\gamma$, and MEK/ERK pathways via VEGFR-2 in a variety of neuronal cells. 

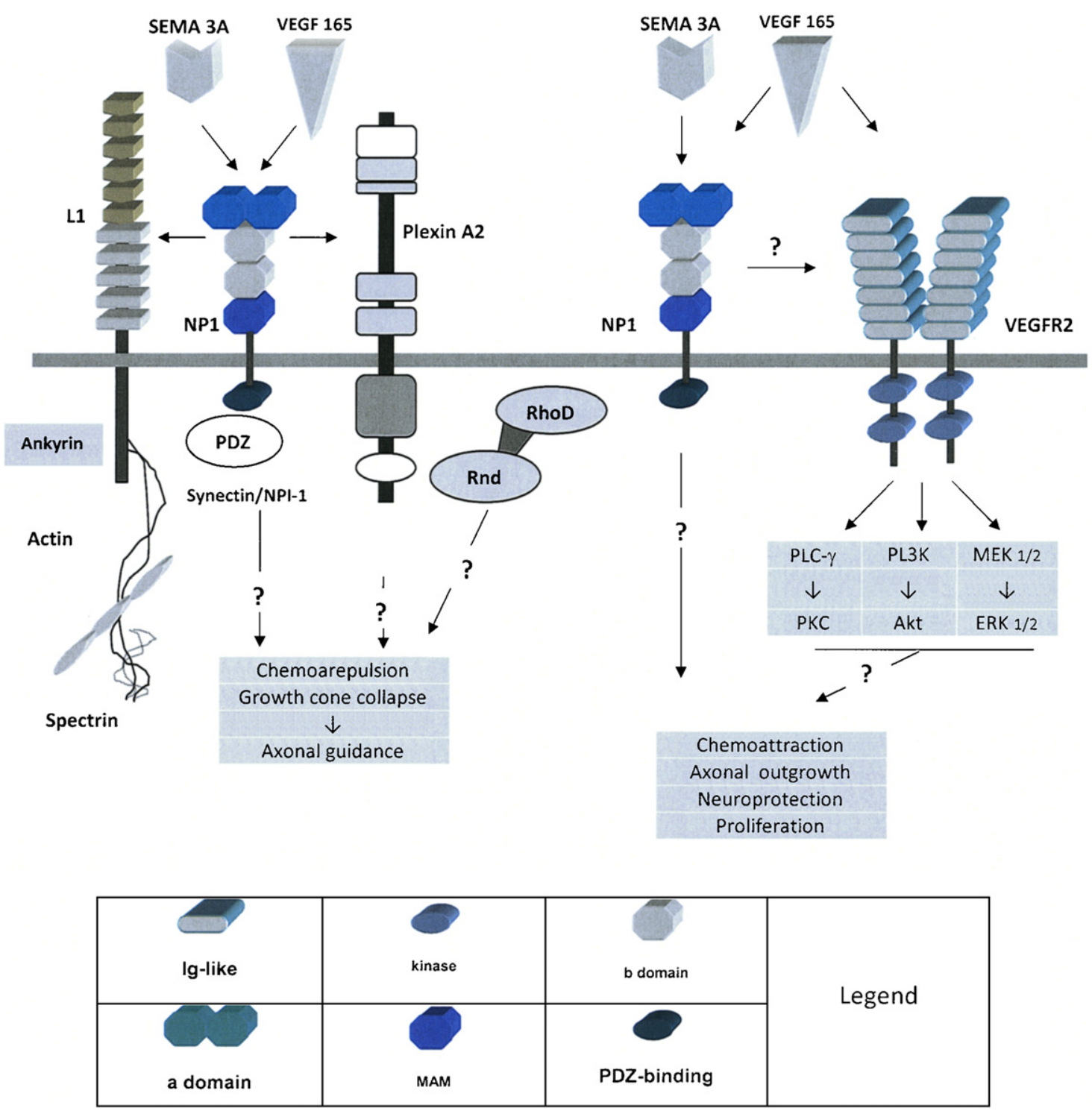

Fig. 2. (Continued).

expressed by endothelial cells, several tumor cell types, and different types of sensory neurons, including dorsal root ganglion, olfactory and optic nerves, as well as some sympathetic neurons. VEGF165 also binds specifically to NP2 that has domain similar to that of NP1 displaying a $44 \%$ of amino acid identity and distinct expression pattern in the developing nervous system. NP2 is also a receptor for the VEGF isoform 145 (Fig. 2B).
VEGF-A and its receptors are of utmost significance for many diseases accompanied by vascular pathology and inflammation, such as cancer stroke, intraocular neovascular syndromes, inflammatory disorders, and brain edema. Although vascular endothelial growth factor receptor-1 (VEGFR-1) has a higher affinity to VEGF-A than VEGFR-2, VEGFR-1 autophosphorylation upon VEGF-A binding is weak in comparison to VEGFR-2. This particular effect 
could be linked to the fine control of VEGF-A activity during development. In fact, early embryonic lethality in knockout (VEGFR-1 -/-) mice has been demonstrated; conversely, mice lacking only the intracellular domain of the VEGFR-1 develop almost normal and reach adulthood. Therefore, it seems that VEGFR-1 mainly functions as a "decoy receptor" during development by regulating VEGF availability. Besides membrane-bound VEGFR-1, a soluble form exists (sFlt-1) that has also the capacity to perform decoy functions. Hence, the VEGF action could be different depending on the dominant isoform produced in a particular district. Therefore, alternative splicing, posttranslational modification, and receptor and antagonistic receptor transduction pathway are the key regulators of this pleiotropic growth and survival factor.

\section{Angiogenesis, mitogenesis, and endothelial survival}

During the development, blood vessels are generated by two processes: vasculogenesis and angiogenesis. Vasculogenesis is the process that defines the de novo formation of blood vessels from undifferentiated endothelial precursor cells. During development, the extra- and intra-embryonic primary vascular plexus, the dorsal aorta, and the primitive heart are formed by vasculogenesis. In angiogenesis, the formation of new blood vessels from preexisting vessels gives rise to a continuous expansion of the primitive vasculature. In these processes, the VEGF-A action is pointed out by knockout mice where the loss of one allele leads to embryonic lethality at midgestation due to defective vasculogenesis in the entire embryo. In addition, the specific inactivation of VEGF-A in brain resulted in growth retardation and neural apoptosis. VEGF-A stimulates proliferation of endothelial cells, and the newly formed blood vessels are guided by VEGF gradient to their target tissues. A specialized, nonproliferating cell at the apical part of a navigating vessel extends filopodia that recognize VEGF-A gradient by its VEGF receptors and guides the vessel toward its target area. Gradients are formed by the higher expression of VEGF in the targeted area and by the different binding capacities of VEGF-A isoforms to the extracellular matrix. The isoforms differ also for the diverse binding capacity to the heparan sulfate proteoglycan and therefore the diffusion within the tissue. VEGF supports in vitro growth of vascular endothelial cells derived from arteries, veins, and lymphatics (Ferrara, 2002). In particular, VEGF promotes angiogenesis in vitro in three-dimensional models, inducing confluent microvascular endothelial cells to invade collagen gels and form capillary-like structures (Pepper et al., 1992) Furthermore, VEGF induces sprouting from rat aortic rings embedded in a collagen gel and also elicits a pronounced angiogenic response in different in vivo models such as the chick chorioallantoic membrane (Leung et al., 1989), stimulates direct angiogenesis in the rabbit cornea (Phillips et al., 1994), is able to induce iris neovascularization, and stimulates direct angiogenesis in the rabbit cornea (Tolentino et al., 1996). VEGF is also a survival factor for endothelial cells, both in vitro and in vivo (Alon et al., 1995). In vitro, VEGF prevents endothelial apoptosis induced by serum starvation. Such activity is mediated by the phosphatidylinositol 3-kinase (PI3K)/Akt pathway (Gerber et al., 1998). Moreover, VEGF induces the expression of the antiapoptotic proteins $\mathrm{Bcl}-2$ and $\mathrm{A} 1$ in vascular endothelial cells. In vivo, the prosurvival effects of VEGF are developmentally regulated. In fact, VEGF inhibition results in extensive apoptotic changes in the vasculature of neonatal, but not adult mice. Furthermore, a strong VEGF dependence has been demonstrated within tumors in endothelial cells of newly formed vessels but not of established ones. Although endothelial cells are the primary targets of VEGF, several studies have reported mitogenic effects also on certain nonendothelial cell types, such as retinal pigment epithelial cells, pancreatic duct cells, and Schwann cells.

\section{Neurotrophic and neuroprotective effect}

Recent evidence suggests that VEGF-A directly affects neurons. In particular, VEGF is a relevant key factor in the developing and in the adult nervous system, displaying a relevant role in angiogenesis, neurogenesis, cell survival, and neuronal migration. During the early development, 
VEGFR-2 is expressed in neural progenitor cells and in some differentiated cells in the developing mouse retina, at this stage still avascular. In this tissue, VEGF-A is expressed concomitantly in VEGFR-2 expressing cell, demonstrating the direct action of VEGF-A and VEGFR-2 in retinal neurogenesis and development (Raab and Plate, 2007). In vitro studies demonstrate that VEGF-A, transducing its action by VEGFR-2, regulates retinal progenitor cell proliferation and neuronal differentiation in chick retina (Hashimoto et al., 2006). The direct effect of VEGF-A on retinal cells was demonstrated in experimental model using isolated cells from newborn rats, by increasing the number of photoreceptors and amacrine cells. Therefore, its action is regulated by the expression of specific receptors alternatively by the action of its antagonistic soluble receptor. An example of this fine regulatory system is the corneal avascularity, required for optical clarity and optimal vision. The molecular basis of corneal avascularity has been for a long time unclear. Recently, it was found that the cornea contains VEGF-A but nearly all of it was bound to sVEGFR-1, also known as sFlt-1, the soluble receptor, recognized as essential factor for preserving the avascular ambit of the cornea.

VEGF-A exerts its action also on axonal outgrowth, on differentiation, and on the survival of superior cervical and dorsal root ganglion neurons and mesencephalic neurons in organotypic explant cultures. In vitro, VEGF-A stimulates axonal outgrowth, improves the survival of superior cervical and dorsal-root ganglion neurons, enhances the survival of mesencephalic neurons in organotypic explant cultures, and can rescue HN33 hippocampal cells from apoptosis induced by serum withdrawal (Jin et al., 2000). In vivo, VEGF-A coordinates the migration of motor neuron soma (Schwarz et al., 2004), whereas local delivery of VEGF-A prolongs motor neuron survival (Storkebaum et al., 2004). Conversely, low VEGF-A levels have been linked to motor neuron degeneration in both animal models and human disease (Oosthuyse et al., 2001).

In addition to a cellular role in regulating proliferation, migration, and permeability, the most recent function assigned to VEGF is that of a neuroprotective agent. A neuroprotective effect has been demonstrated also in various brain diseases: Parkinson's disease, stroke, and amyotrophic lateral sclerosis (ASL). The neurotrophic and neuroprotective effect of VEGF-A is the result of the complex cross talk among different soluble factors and involves a combination of direct neuroprotective effects and stimulation of angiogenesis, implying an enhanced supply of oxygen and nutrients that indirectly exert a neuroprotective impact.

Therefore, VEGF-mediated neuroprotection in the central nervous system has been attributed to both indirect and direct effects of VEGF-A on neuron survival. VEGF exerts neuroprotective actions indirectly through multiple mechanisms such as stimulation of angiogenesis, increasing blood flow, and enhancing brain barrier permeability for glucose and antioxidant activation (Góra-Kupilas and Josko, 2005).

Furthermore, it has been demonstrated that VEGF-A has a direct survival effect on neuronal cells of the retina, independent of blood flow, and functional analyses showed that VEGFR-2, detected in several neuronal cell layers of the retina, was involved in retinal neuroprotection.

Mechanisms implicated in direct neuroprotection involve the inhibition of the programmed cell death of neuronal cells under hypoxic conditions and stimulation of neurogenesis.

Under critical conditions for neuronal cells (hypoxia, glucose deprivation, oxidative stress), VEGF-A becomes a mediator of multiple molecular reactions leading to the inhibition of programmed cell death-inducing Bcl-2 antiapoptotic factor, inhibiting the activation of the proapoptotic factor Bax, and the stimulating neurogenesis (Góra-Kupilas and Josko, 2005). A significant regulatory step of apoptosis is the activation of the proapoptotic factor Bax. The overexpression of Bcl-2 induced by VEGF-A inhibits the activation of Bax. The mechanism by which Bax activation is regulated is still unclear, although several downstream events have been elucidated.

Following its activation, Bax homodimerizes and translocates into the mitochondria membrane, conferring permeability and leading to the release of several death-promoting factors (cytochrome $c$ ) in the cytoplasmic compartment. Therefore, the regulation of its activation is clearly relevant in 
normal cell survival under physiological conditions and still conflicting in tumor insurgence as well as in tumor progression. Recently, some information was published on its activity regulation. Bax is localized physiologically inactive in the cytoplasm in normal undamaged cells interacting with the $\mathrm{Ku} 70$ protein $\mathrm{C}$ terminus; this status determines its inability to homodimerize and give rise to apoptotic key events. Overexpression of Ku70 in vitro blocks the Bax-induced apoptosis under some variety of stimuli in epithelial cells (Leskov et al., 2003). Following UV-induced DNA damage, the DNA double-strand breaks sensor Ku70 translocates to the nucleus, allowing Bax release and its translocation to the mitochondria. This important function of $\mathrm{Ku}$ as regulator of Bax-mediated releases of several death-promoting factors is in agreement with its role as caretaker in the nucleus. Microenvironmental factors, such as VEGF or IL6 , are strictly connected to the $\mathrm{Ku} 70-$ Bax-binding induction to prevent Bax activation and translocation in the mitocondria (Sawada et al., 2003). This scenario strongly influences cell survival increasing Bcl-2, Bax antagonist, when the cell death program is redirected into survival (Pucci et al., personal communication). These molecular interactions are still obscure, but underline the relevant role of VEGF and other microenvironmental factors in the complicated cross talk among molecules that could effectively turn the cell fate.

Moreover, VEGF exerts a direct effect on neurons, as demonstrated in vitro. VEGF-A has a neurotrophic effect on cultured neural cells of the peripheral and central nervous systems by stimulating the axonal outgrowth and by protecting neural cells from serum deprived-glutamateinduced or hypoxia-induced cell death. The neuroprotective function of VEGF includes also the indirect actions that affect neuronal survival under critical conditions and may be related to (i) increased blood flow and oxygen supply, (ii) enhanced endothelial permeability with increased glucose transport across blood-brain barrier (BBB), (iii) the antioxidative function of VEGF and heme oxygenases, and (iv) angiogenesis.

i. It has been demonstrated that VEGF-A can increase tissue blood flow via nitric oxide induction and that increased flow (and oxygen supply) in many different ischemic settings has been shown to preserve tissue integrity and function (Endres et al., 2004). In a model of ischemia-reperfusion injury (I/R injury model), it has been demonstrated that VEGF-A injection can increase volumetric blood flow also in the retina provoking a dose-dependent reduction in retinal neuron apoptosis. The role of the incremental increase in blood flow on neuroprotection was assessed by inhibiting the activity of iNOS in the I/R injury model, which led to a significant reduction (but not complete suppression) of the neuroprotective effects of VEGF-A, suggesting that VEGF-A-induced volumetric blood flow to the retina may be at least in part responsible for the neuroprotection (Nishijima et al., 2007).

ii. Glucose, the most important energetic substrate for the brain, must pass the BBB to obtain access to the brain. There are studies indicating VEGF as a candidate for directly regulating brain glucose uptake under critical conditions (Dorle et al., 2002).

iii. In fact, VEGF mediates the enhancement of glucose transport through the $\mathrm{BBB}$ by increasing GLUT-1 gene expression and translocation of cytosolic GLUT-1 to the plasma membrane surface. Furthermore, VEGF mediates induction of endothelial fenestrations, thereby increasing the transport of small molecules (Sone et al., 2000). In conclusion, VEGF can increase brain glucose uptake by enhancing the transport via GLUT-1, dilating cerebral arterioles, and inducing endothelial fenestrations under conditions of brain glucose deprivation.

iv. Moreover, VEGF-A also exerts its action on oxidative stress. Human heme oxygenase (HO) exists in three isoforms: HO-1 (inducible), HO-2, and HO-3 (constitutive). HO-1 is widely distributed and induced by a range of stimuli including shear stress, oxidative stress, nitric oxide (NO), and hypoxia (Maines, 1997). HO-1 is a cytoprotective molecule that acts, in the presence of a variety of noxious stimuli, to exert antiapoptotic, 


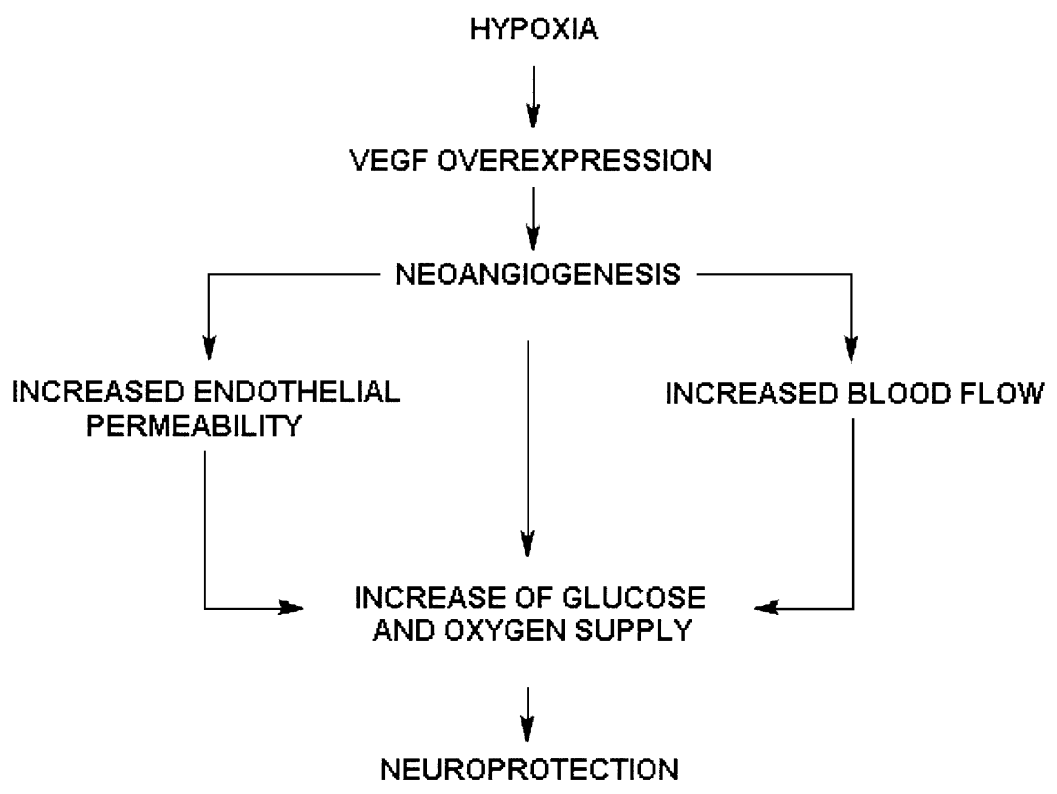

Fig. 3. Indirect neuroprotective effect of VEGF. VEGF regulates brain glucose uptake under critical conditions.

anti-inflammatory, and antiproliferative effects. HO-1 is rapidly induced during hypoxia, ischemia-reperfusion, hyperthermia, and endotoxic shock, providing cytoprotection during the resolution of stress-induced inflammatory injury (Fig. 3).

v. Recent in vivo studies report that VEGF regulates HO-1 expression and activity in vascular endothelial cells. The inhibition of HO-1 abrogates VEGF-induced endothelial activation and subsequent angiogenesis but promotes VEGF-induced monocyte recruitment and inflammatory angiogenesis. HO-1 may also regulate the synthesis and activity of VEGF, resulting in a positive-feedback loop (Józkowicz et al., 2003). HO-1 participates in heme degradation. Carbon monoxide (CO), ferrous ions $\left(\mathrm{Fe}^{2+}\right)$, and biliverdin are the products of this reaction, which prevent cells from oxidative damage caused by free radicals. Ferritin, biliverdin, and bilirubin are physiological antioxidants in serum and extravascular space, which protect neurons from damage due to the production of reactive oxygen species. Hypoxia by inducing HOs contributes to the increase in the concentration of heme degradation products. These products exert their neuroprotective actions through vessel dilatation and antioxidation. Moreover, HO-1 induces expression hypoxia-inducible factor- $1 \alpha$ (HIF- $1 \alpha$ ), including the gene encoding VEGF. VEGF induces backward HO-1 and, through this positive biofeedback, it mediates in antioxidation (Fig. 4) (Motterlini et al., 2000).

vi. Many studies on cerebral ischemia in animals reveal that hypoxia induced by occlusion of the cerebral middle artery contributes to overexpression of VEGF and angiogenesis. Other observations on rats show that overexpression of VEGF occurs also after subarachnoid hemorrhage (SAH). Increased expression of this factor is particularly detected in some regions of the brain hippocampus, thalamus, lateral ventricles, the fourth ventricle, smooth muscle cells of subarachnoid vessels, and cerebellar cortex. The potential, therapeutic usefulness of VEGF in cerebral ischemia is also limited by the fact that its angiogenic effect is delayed in onset and, therefore, presumably too late to rescue many vulnerable neurons. The 


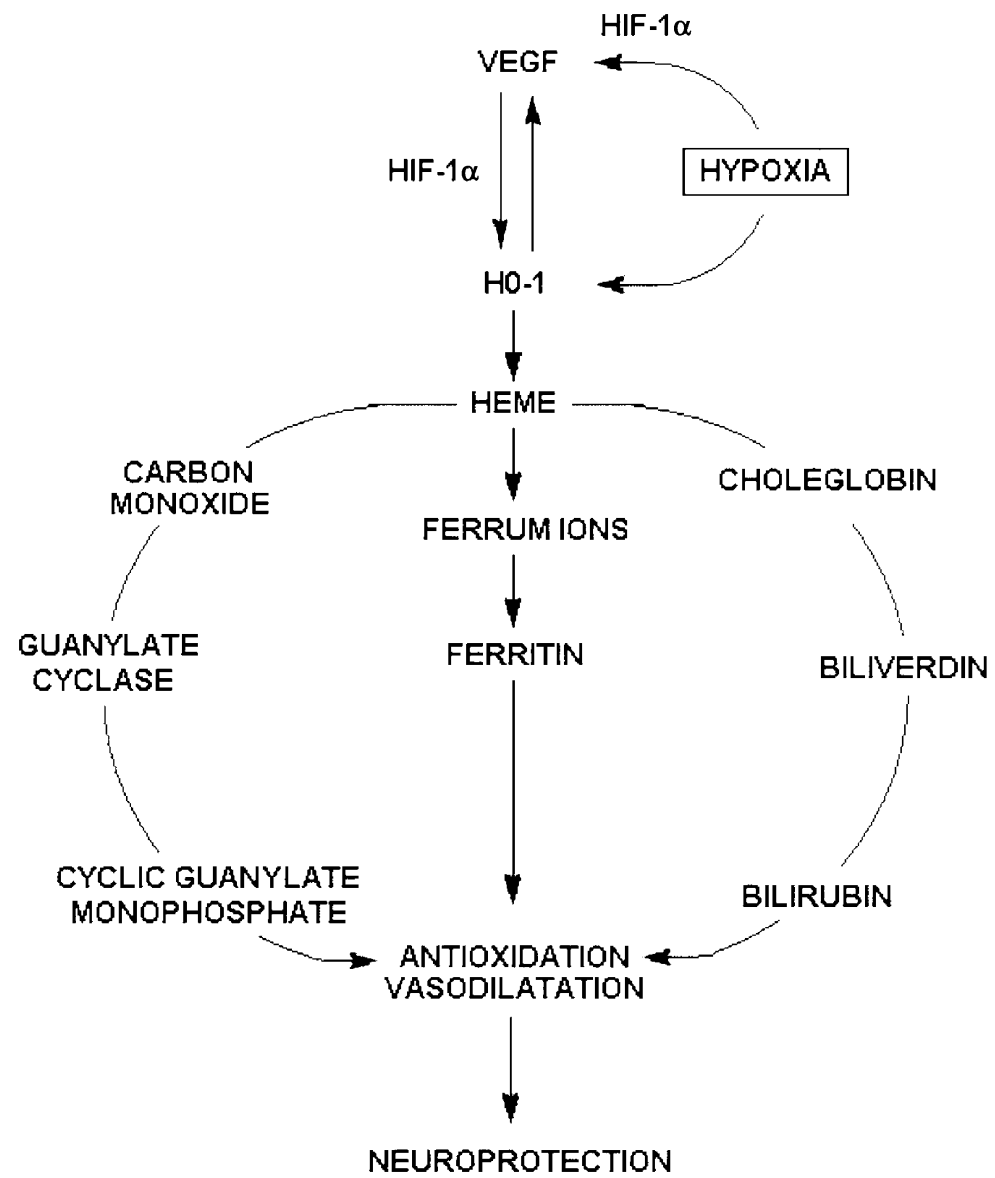

Fig. 4. Effect of VEGF on the oxidative stress and its downstream cascade.

possibility of direct neuroprotective VEGF actions in ischemic tissue in the interval preceding angiogenesis may help prolong cell survival until angiogenesis can occur.

VEGF neuroprotection is exerted mainly by the activation of the VEGFR-2. Additional evidence for direct neuronal protection by VEGF is that VEGF inhibits the death of cultured hippocampal neurons from glutamate and $N$-methyl-d-aspartate toxicity and that deletion of the hypoxia-response element from the VEGF promoter causes motor neuron degeneration in mice.

Because neuroprotective effects of other growth factors are associated with improved outcome from stroke, this could be true for VEGF as well. Finally, VEGF has been implicated as a factor that promotes neurogenesis in the adult brain. One possibility is that this occurs through the establishment of a vascular niche that favors the proliferation and differentiation of neuronal precursors, perhaps by the release of BDNF from endothelial cells. Alternatively, VEGF may exercise a direct mitogenic effect on neuronal precursors.

Thus, VEGF expression in the ischemic brain could contribute to ischemia-induced neurogenesis and modify outcome in that way as well.

\section{Intravitreal VEGF inhibition therapy and neuroretina toxicity}

The neuroprotective function of VEGF-A in the retina has not been characterized despite the fact 
that VEGF antagonists are being applied widely to combat retinal vascular disease. A recent study compared the antiproliferative and cytotoxic properties of bevacizumab (Avastin), pegaptanib (Macugen), and ranibizumab (Lucentis) on human retinal pigment epithelium (ARPE19) cells, rat retinal ganglion cells (RGC5), and pig choroidal endothelial cells (CECs). Ranibizumab reduced CEC proliferation by $44.1 \%$, bevacizumab by $38.2 \%$, and pegaptanib by $35.1 \%$ when the drugs were used at their established clinical doses. However, the differences between the three drugs in respect to cell growth inhibition were not statistically significant. Only a mild antiproliferative effect of bevacizumab or pegaptanib on ARPE19 cells could be observed. Ranibizumab did not alter ARPE19 cell proliferation. No cytotoxicity on CEC, RGC5, and ARPE19 cells could be seen. In this view, bevacizumab, pegaptanib, and ranibizumab significantly suppress CEC proliferation. However, when used at the currently established doses none of the drugs was superior over the others in respect to endothelial cell growth inhibition. The biocompatibility of all three drugs — including the off-label bevacizumab - seems to be excellent when used at the currently recommended intravitreal dose (Spitzer et al., 2007).

\section{Neuroprotection: clusterin, a multifunctional protein}

\section{Clusterin/ApoJ: a debated physiological role}

Clusterin/Apolipoprotein $\mathrm{J}(\mathrm{Clu} / \mathrm{ApoJ})$ is a heterodimeric, highly conserved, secreted glycoprotein being expressed in a wide variety of tissues and found in all human fluids. The protein has been reportedly implicated in several diverse physiological processes such as sperm maturation, lipid transportation, complement inhibition, tissue remodeling, membrane recycling, cell-cell and cell-substratum interactions, and promotion or inhibition of apoptosis.

The human clusterin comprises 449 amino acids, generating an unglycosylated holoprotein (precursor form, pCLU) with a predicted molecular mass of $60 \mathrm{kDa}$ that can be proteolytically cleaved into $\alpha$ - and $\beta$-subunits held together by disulfide bonds.
Mature clusterin is glycosylated and secreted as a protein of 76-80 kDa (Wong et al., 1993; Lakins et al., 1998) depending on the degree of glycosylation, which appears like a $40-\mathrm{kDa} \alpha$ - and $\beta$-protein smear, by polyacrylamide gel under reducing conditions (Leskov et al., 2003). Its high degree of sequence conservation, almost ubiquitous tissue distribution, and the absence of functional clusterin polymorphisms in humans suggest that the protein accomplishes a function of fundamental biological importance.

Two different mRNA transcripts for clusterin, derived from an alternative splicing, have been identified, one coding for the secreted form of clusterin $(40 \mathrm{kDa})$ and the other one coding for the 50- to 55-kDa nuclear form, without leader peptide. The presence of these two different isoforms and their function within the cell is a much-debated question. One form was found expressed preferentially in the nucleus (nClu) of different cell types, while the highly glycosylated form (sClu) was found in the cytoplasm and secreted in biological fluids. The activation of nuclear clusterin form seems to induce cell cycle arrest and cell death. Leskov et al. (2003) demonstrated that clusterin nuclear form is unglycosylated and is involved in apoptosis induction. Its function is related to the activation of $\mathrm{Ku} 70$ and Bax cascade as previously described as one of VEGF antiapoptotic direct functions. In normal cells after irreversible cell damage, nClu cooperates with $\mathrm{Ku} 70$ to induce apoptotic death activating Bax translocation to mitocondria. In tumors, its expression is inhibited favoring the presence of the antiapoptotic form $(\mathrm{sClu})$ that inhibits cell death and favors the aberrant neoplastic cell clone expansion. In fact, it has been demonstrated that the overexpression of the secreted isoforms in the cytoplasm correlated with the apoptotic cell death resistance (Leskov et al., 2003; Pucci et al., 2004). Paradoxically, cell survival and cell death are finely regulated by the balanced expression of different isoforms of the same protein and by the intriguing pathways of growth- or death-promoting factors of the microenvironment.

In response to cellular stress conditions, including heat shock, UV radiation, and oxidative stress, 
and in a wide array of pathological conditions, such as neurodegenerative disorders, multiple sclerosis, atherosclerosis, myocardial infarction, and cancer, clusterin expression is markedly upregulated in vitro and in vivo. Evidence suggests that sClu exerts a cytoprotective function under these stress conditions, which may be mediated by protection against oxidative stress (i), inhibition of apoptosis and complement-mediated cell lysis (ii, iii), promotion of cellular contacts (iv), and protection of cell membranes (v) (Zenkel et al., 2006).

\section{Clusterin and diseases}

Clusterin gene is differentially regulated by cytokines, growth factors, and stress-inducing agents; upregulation of clusterin mRNA and protein levels is also detected in neurodegenerative conditions related to aging (Trougakos and Gonos, 2002). nClu accumulates during the growth-arrested cellular state of senescence that is thought to contribute to aging and suppression of tumorigenesis (Kyprianou et al., 1991; Bettuzzi et al., 2002); however, the secreted isoform sClu is also upregulated in several cases of in vivo cancer progression and tumor formation (Pucci et al., 2004). Pucci et al. (2004) have demonstrated that the progression toward high-grade and metastatic colorectal carcinomas correlates to the overexpression of the glycosylated cytoplasmic form of clusterin (sClu) and to a complete loss of the proapoptotic nuclear form (nClu). Thus, the increased level of the secreted form and the disappearance of the nuclear unglycosylated one are directly connected to increased cell survival, aggressiveness, and enhanced metastatic potential.

Therefore, clusterin can act either to promote or to inhibit cell death, depending on the cellular context or molecular species. The question of whether clusterin is a multifunctional protein, or deploys a single primary function influenced by cellular context, remains a central issue continuing to stimulate interest in this enigmatic molecule (Jones and Jomary, 2002).

More recently, it has been proposed that clusterin represents a new class of highly efficient chaperones, similar to the small heat-shock proteins; however, the heat-shock proteins act in the intracellular space, whereas for sClu the main site of action is the extracellular space. Molecular chaperones serve as a cellular rescue system by interacting with partially folded or unfolded stressed proteins to prevent their aggregation and precipitation and to promote protein refolding. Misfolding of proteins is a result of structural modification typically caused by oxidative stress or mutation. sClu binds to exposed hydrophobic regions of a broad range of partially unfolded proteins producing solubilized high-molecularweight complexes, thereby preventing their aggregation and precipitation (Poon et al., 2000; Wilson and Easterbrook-Smith, 2000). Moreover, sClu has been shown to preferentially interact with slowly aggregating target proteins (Poon et al., 2002). Clusterin does not affect the refolding of destabilized proteins, but it stabilizes them in a state competent for refolding into functional proteins by other chaperones (such as heat-shock protein 70).

\section{Clusterin and the nervous system}

The normal adult brain is a major site of Clu/ApoJ mRNA synthesis in several mammalian species (Collard and Griswold, 1987; Duguid et al., 1989). High levels of clusterin mRNA were found in several neuron-rich layers and nuclei, including the pyramidal and granular cell layers of the hippocampal formation, the hypothalamus, and some motor neurons (Danik et al., 1999). In the cerebral cortex, a homogeneous pattern of hybridization was observed in which neurons as well as astrocytes expressed clusterin mRNA. In contrast with the high levels and ubiquitous distribution of clusterin mRNA, only a few structures showed clusterin immunoreactivity by immunohistochemistry (Pasinetti et al., 1994; Calero et al., 2000). Overall, we can distinguish two general mechanisms exerted by clusterin that could induce a direct neuroprotection, through the triggering of signaling cascades that affect neurodegenerative pathways and apoptotic processes, and an indirect neuroprotection, through (i) its interaction with amyloid- $\beta(\mathrm{A} \beta)$ peptides, by its role of chaperone; (ii) the inhibition of the formation of the 
membrane-attack complex of the complement system; and (iii) neurotrophic functions, promoting a general recovery from neuronal injury via lipid transport or membrane recycling.

In the Alzheimer's disease (AD), the most frequent form of amyloidosis in humans and the major cause of dementia in the elderly, clusterin is found associated with $A \beta$ plaques and cerebrovascular deposits. Clusterin coprecipitates with $A \beta$ from cerebrospinal fluid, suggesting a physiological interaction with $\mathrm{A} \beta$. The presence of sClu in parenchymal and vascular amyloid deposits may result as a consequence of the inflammatory process and complement activation occurring in the lesions rather than constituting a specific mechanism for the disease. Current knowledge suggests that $\mathrm{sClu}$ has a protective function in $\mathrm{AD}$ : mammalian recombinant clusterin obtained from stable transfection of hamster kidney fibroblasts as well as a commercial native clusterin, purified from serum, provides dose-dependent neuroprotection against A $\beta$ (Boggs et al., 1996). Thus, the interaction of clusterin with $A \beta$ could modulate $\mathrm{A} \beta$ neurotoxicity. Furthermore, in primary neuronal cell cultures, clusterin prevents $A \beta$ toxicity (Calero et al., 2000).

In biological fluids, clusterin maintains sA $\beta$ solubility and modulates its uptake by the brain across the BBB. Moreover, clusterin promotes the $\mathrm{A} \beta$ catabolism through the formation of soluble complexes that may follow catabolic pathways common to the lipoprotein particles.

As reported above, clusterin is markedly induced after tissue injury. In the nervous system, clusterin upregulation may represent a defense response against local nerve damage. Moreover, as a chaperone protein, clusterin could be involved in the protection of the nervous system from potential toxic debris.

Dati et al. (2007) have investigated the effects of recombinant human clusterin (r-h-clusterin) in different experimental models of peripheral neuropathies. Daily treatment with clusterin accelerated the recovery of nerve motor-evoked potential parameters, after nerve injury. Treatment of experimental autoimmune neuritis rats with clusterin accelerated the rate of recovery from the disease, associated with remyelination of demyelinated nerve fibers. These data demonstrate that clusterin is capable of ameliorating clinical, neurophysiological, and pathological signs in models of peripheral neuropathies. Taking into account the interplay between inflammation and neurodegeneration in the peripheral nervous system, the effects of r-h-clusterin could be both immunomodulatory and neurotrophic on myelinating Schwann cells. Recombinant clusterin may prevent apoptosis in these experimental models. These authors concluded that r-h-clusterin does not exert immunomodulatory effect, based on the absence of significant changes in blood and nerve cytokine concentrations or in situ macrophage infiltration. However, r-h-clusterin could modify the cytokine-network abnormalities known to occur in inflammatory neuropathies. For example, it has been known that transforming growth factor- $\beta 1$ (TGF- $\beta 1$ ) controls clusterin expression (Morgan et al., 1995). This observation emphasizes the importance of early modulation of the inflammatory microenvironment.

Interestingly, investigations of knockout $(\mathrm{Clu}-/-)$ mice have complicated the picture, highlighting the two faces of clusterin. On the one hand, absence of clusterin reduces cell death in hypoxia-ischemiainduced brain damage, suggesting that it normally functions to exacerbate neuronal damage in these circumstances (Han et al., 2001), whereas in the same knockout strain, autoimmune myocardial damage is increased, implying a normally protective role (McLaughlin et al., 2000). Moreover, inducing brain injury by middle cerebral artery occlusion in clusterin gene knockout ( $\mathrm{Clu}-/-)$ mice identical to those used by Han et al., in transgenic mice overexpressing clusterin and wild-type mice, Wehrli et al. (2001) demonstrated a $30-50 \%$ increase in clusterin mRNA levels in the ischemic mouse brains, as compared with the control hemisphere of wild-type mouse brains. This area sustains the inflammation and contains cells that retain the potential for salvage. At 14 days post ischemia, wild-type mice displayed a much higher number of inflammatory cells than clusterin-overexpressing mice, and $\mathrm{Clu}-/-$ mice showed the worst morphological resolution. Overall, an inverse correlation between clusterin expression and ischemic brain damage was observed, showing that clusterin 
protects against ischemic brain damage (Wehrli et al., 2001).

Cortical spreading depression (CSD) is a powerful benign stimulus that is capable of providing long-lasting ischemic tolerance. It is classically characterized as a slowly propagating wave $(1-6 \mathrm{~mm} / \mathrm{min})$ of neuronal and astrocytic depolarization that results in transient suppression of electroencephalographic activity. According to previous studies that have implicated clusterin as a protective molecule assisting in neuronal survival, the clusterin mRNA levels significantly increase 1-14 days after CSD. These findings, along with other studies in clusterin-transgenic mice, suggest that increased clusterin production and secretion, particularly by astrocytes, could play a role in CSD-induced conditioning/protection of cortex, perhaps via one or more of its actions, such as inhibition of complement activation and cytolysis, effects on chemotaxis and apoptosis, or as an antistress protein chaperone (Wiggins et al., 2003).

Moreover, HIF- $1 \alpha$ activation, influenced by VEGF-A, acts on clusterin gene induction and could be directly related to the preferential clusterin isoforms produced. Moreover under stress conditions, many stress-derived factors and cytokines (i.e., IL-6, TGF- $\beta$ ) could induce this prosurvival protein, as demonstrated by another enigmatic link among clusterin, the function of VHL and its target HIF (Nakamura et al., 2006). Therefore, sClu could be induced in the presence of HIF- $1 \alpha$, condition necessary but not sufficient for its production.

\section{Neuroprotection: IL-6, VEGF, clusterin, and glaucoma}

Glaucoma represents a group of neurodegenerative diseases characterized by anatomical damage to the optic nerve and slow, progressive death of RGCs.

Elevated intraocular pressure (IOP) is considered to be the most important risk factor for glaucoma, and treatment options for the disease have been traditionally limited to its reduction. However, visual field loss and RGC death continue to occur in patients with well-controlled IOP normal tension glaucoma and thus the concept that treatment strategies alternative to IOP lowering are needed has recently emerged.

This concept emphasizes that several pressureindependent mechanisms are involved in the development and progression of glaucomatous neuropathy and that high IOP and optic nerve vascular deficiency could be necessary but not sufficient in the pathogenesis of glaucomatous damage.

An advocated strategy concerns the development of neurotrophic agents that would interact with neuronal or glial elements within the retina and/or optic nerve head allowing the RGCs survival.

The advent of animal models of chronic glaucoma has greatly enhanced the understanding of many of the processes occurring in glaucoma pathogenesis and addressed "theoretical" targets for pharmacological intervention. Such targets include glutamate receptors, autoimmune elements, neurotrophin deprivation, nitric oxide synthesis, oxidative stress products, sodium and calcium channels, heat-shock proteins, and apoptotic pathways.

For example, the death of RGC could be the result of a biphasic process: a primary injury responsible for damage induction that is followed by a slower secondary degeneration, related to noxious environment surrounding the degenerating cells. Retinal ischemia may provoke a cascade of events that result in cell death: hypoxia leads to cytotoxic levels of glutamate that cause a rise in intracellular calcium, which in turn leads to neuronal death, due to apoptosis or necrosis. Neuroprotective agents would attempt to protect the cells that were spared during the initial insult but are still vulnerable to environmental damage.

Many downstream factors are involved in neuroprotection after damage or inflammatory disorders. Usually, for example, IL-6 involved in some pro-inflammatory diseases or tumorigenesis is retained as a negative prognostic factor, but the presence of this cytokine in the acute phase of cerebral ischemia plays a critical role in preventing damaged neurons from undergoing apoptosis and its role may be mediated by Stat 3 activation. 
Moreover, IL-6 determines an increased neuroprotection inducing VEGF, clusterin, and hypoxia- $1 \alpha$ activation.

The increased amount of this pro-inflammatory cytokine after neuronal damage orchestrates the cascade of events that turn death into survival.

IL-6, which has been identified as B-cellstimulating factor, belongs to the subfamily of cytokines, including leukemia inhibitory factor and ciliary neurotrophic factor (CNTF) that share the gp130 subunit as a common receptor subunit.

IL- 6 binds to IL- 6 receptor $\alpha$ that is composed of a membrane-bound and soluble form. The association of the IL-6/IL-6 receptor $\alpha$ with gp130 causes the dimerization of gp130, followed by the activation of the Janus kinase-signal transducer and activator of transcription signal (JAK-STAT) pathway. In addition, gp130 in turn activates the extracellular signal-regulated kinase (ERK) and the PI3K/Akt pathway.

Clinical studies on ischemic stroke have previously demonstrated a correlation between IL-6 serum levels, body temperature, expression of inflammatory proteins (C-reactive protein), infarct volume, and severity of paresis. Previous studies in rat brain and glia demonstrated that IL-6 is expressed by neurons and glia. Neuroprotective effect of exogenous IL-6 has been observed in PC12 cell cultures in a hypoxia-reoxygenation paradigm, in peripheral nerves after trauma, and in ischemic neurons. Although the role of IL-6 in stroke is still a matter of controversy, identifying extracellular signals that modulate RGC survival in glaucoma and determining whether these signals depend on pressure are essential for delineating the mechanisms of disease and for defining new targets for its treatment. Astrocytes and microglia represent a source of these signals.

The concept that the glia in the optic nerve could influence the survival of RGCs in glaucoma is widely accepted; however, the contribution of the retinal glia to pathological processes associated with glaucoma is less clear. Thus, glia-derived IL-6 could modulate RGC survival in glaucoma, particularly in the retina, where astrocytes and microglia are in proximity to RGC somas and axons. Astrocytes and microglia are subjected to elevated pressure, affecting the survival of RGCs, and IL-6 is a key component of microglial response to elevated pressure serving as a neuroprotectant for RGCs challenged by pressure (Orellana et al., 2005; Yamashita et al., 2005).

In addition to IL-6 itself, proteins related to IL-6, such as CNTF and neuropoietin, may also influence the development and survival of retinal cells. Many antiapoptotic and survival factors are induced by IL-6. Overall, the presence of IL-6 induced VEGF by the activation of nuclear factorkappa B (NF- $\mathrm{B})$ and activator protein 1 (AP-1). The AP-1 activation resulted in the increased expression of both, VEGF and IL-6 regulated in paracrine and in autocrine fashion. Concomitantly, it was found that increased levels of IL-6 and VEGF are closely linked to increased nuclear protein levels of HIF- $1 \alpha$ and enhanced nuclear transcription factor DNA-binding activity to a hypoxia-inducing element located in the VEGF promoter. The characterization of the VEGF165 and VEGF120 variants is a relevant improvement in the discovery of the regulatory pathway of this abundant growth factor. In particular, the neuroprotective action of VEGF120 has been reported as a survival factor for retinal neurons and a critical neuroprotectant during adaptive response to ischemic injury (Nishijima et al., 2007). It resulted that treatment with VEGF might provide neuroprotection in the retina, particularly during ischemic diseases. The use of receptor-specific ligand confirmed that VEGF2 activation is sufficient to trigger retinal neuroprotection and the role of the NP-1 coreceptor appears to be not essential in mediating neuroprotection effects. In fact, blocking VEGF165 with pegaptanib, which does not bind to VEGF120, there is no decrease in retinal RGC viability. As VEGF120 does not bind to NP1, it is unlikely that NP1 is required for this effect. These data demonstrated the important role of VEGFR-2 bound in transducing signals related only to a specific function of this neuroprotectant factor.

Concomitantly to the releases of VEGF in stress condition, there is also the activation of HIF-1 $\alpha$ factor that leads to cascade of prosurvival pathways. Although the role of HIF- $1 \alpha$ in glaucoma was rarely examined due to the problems of collecting intraocular samples from the retina and 
the optic nerve head, there is little evidence to demonstrate hypoxia in the human glaucomatous retina (Tezel and Wax, 2004). Some data on immunolocalization of cellular HIF- $1 \alpha$ in donor eyes from cadaver patients with a diagnosis of glaucoma were obtained by immunoperoxidase and doubleimmunofluorescence experiments. These data showed that the immunostaining for HIF- $1 \alpha$ in the retina and optic nerve head of glaucomatous eyes was higher than that in control eyes, the immunostaining being most prominent in the retinal regions corresponding to the areas of decreased light sensitivity, as determined by visual field testing. Interestingly, HIF- $1 \alpha$ could be localized by immunostaining from the eyes of patients with normal tension glaucoma with a similar spatial relationship with visual field damage (Tezel and Wax, 2004). Further studies are warranted, revealing a possible role for HIF- $1 \alpha$ in the neuroprotection of the glaucomatous retina (Naskar and Dreyer, 2001).

The presence of network of shared neuroprotectant pathways seems to be due to IL-6 that orchestrates the connection among prosurvival factors. Hence, IL-6 induces VEGF that directly influences HIF- $1 \alpha$ activation and clusterin antiapoptotic isoform production (sClu), Bax activity inhibition and Bcl-2 overexpression: exactly as a tumor cell behaves to survive (Fig. 5).

In the experimental model of the oxidative stress, neuroprotection may be mediated by IL-6 via its activation of gp130, the transmembrane

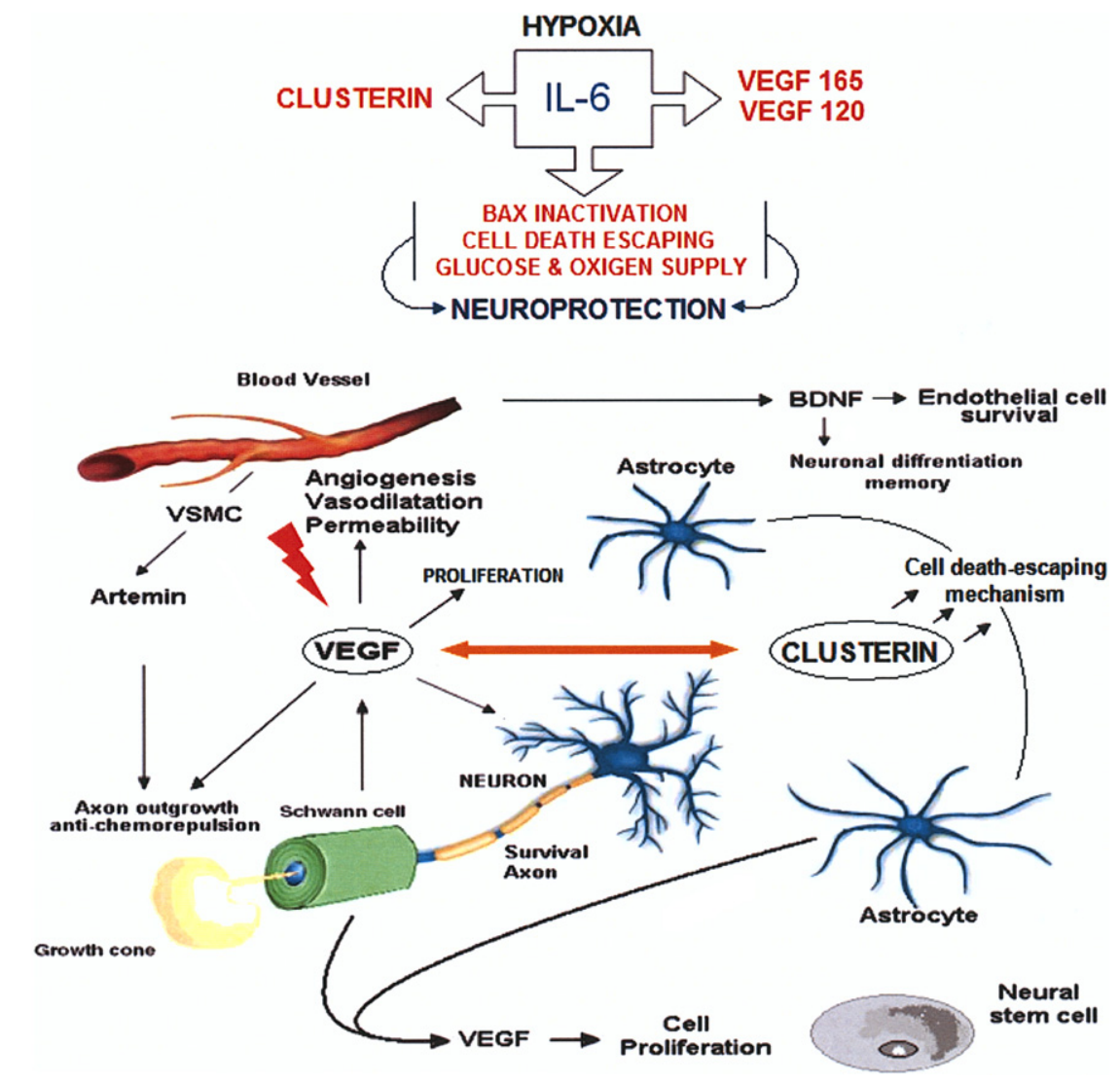

Fig. 5. Downstream factors involved in neuroprotection after damage or oxidative stress. IL-6 is a key component of survival especially in microglial response to elevated pressure. The induction of IL-6 after stress orchestrates the survival pathways. The IL-6 release induces the expression of VEGF that activates HIF- $1 \alpha$ and the prosurvival sClu. The overexpression of sClu and the disappearance of the proapoptotic nuclear isoform ( $\mathrm{nClu}$ ) cooperate with $\mathrm{Ku} 70$ to inhibit Bax-dependent cell death. 
glycoprotein involved in many cytokine-mediated responses (Van Lenten et al., 2001). This experimental model, mimicking the oxidative stress, demonstrated a direct connection between IL-6 production and the increased clusterin mRNA levels, as we have found in colon tumorigenesis (Pucci et al., 2000, and confidential data). Therefore, the important aspect of secreted clusterin as antiapoptotic stress folding protein in this context assumes a key role in neuronal cell survival.

In fact, clusterin plays an important role as a neuroprotectant agent, during different sources of cellular stress as an antiapoptotic signal. In particular, the cytoplasmic glycosylated isoform would play the antiapoptotic role, promoting cell survival, displaying a protection against oxidative stress; inhibiting the membrane-attack complex of complement proteins, locally activated as a result of inflammation (Jenne and Tschopp, 1989); and binding to hydrophobic regions of unfolded, stressed proteins, and therefore avoiding aggregation in a chaperone-like manner (Poon et al., 2000). Although there is good laboratory evidence for glaucoma neuroprotection by several drugs, proof of clinical efficacy for any neuroprotective agent has not yet been obtained in glaucoma. As a consequence, new drugs are needed for testing in appropriate randomized clinical trials for glaucoma neuroprotection. This striking result on clusterin, VEGF-A120, and VEGF-A165 suggests that future therapeutic strategies may need to refocus on the challenge of normalizing rather than abrogating VEGF-A responses.

Taking into account all of the experimental evidences reported above about the role of clusterin, VEGF-A120, and VEGF-A165 in enhancing neuronal survival and in decreasing or preventing neuronal damage, these factors could be considered as new potential glaucoma neuroprotective agents.

\section{References}

Alon, T., Hemo, I., Itin, A., Pe'er, J., Stone, J. and Keshet, E. (1995) Vascular endothelial growth factor acts as a survival factor for newly formed retinal vessels and has implications for retinopathy of prematurity. Nat. Med., 1: 1024-1028.
Bates, D.O., Cui, T.G., Doughty, J.M., Winkler, M., Sugiono, M., Shields, J.D., Peat, D., Gillatt, D. and Harper, S.J. (2002) VEGF(165)b, an inhibitory splice variant of vascular endothelial growth factor, is down-regulated in renal cell carcinoma. Cancer Res., 62: 4123-4131.

Bettuzzi, S., Scorcioni, F., Astancolle, S., Davalli, P., Scaltriti, M. and Corti, A. (2002) Clusterin (SGP-2) transient overexpression decreases proliferation rate of SV40-immortalized human prostate epithelial cells by slowing down cell cycle progression. Oncogene, 21(27): 4328-4334.

Boggs, L.N., Fuson, K.S., Baez, M., Churgay, L., McClure, D., Becker, G. and May, P.C. (1996) Clusterin (Apo J) protects against in vitro amyloid- $\beta(1-40)$ neurotoxicity. J. Neurochem., 67(3): 1324-1327.

Calero, M., Rostagno, A., Matsubara, E., Zlokovic, B., Frangione, B. and Ghiso, J. (2000) Apolipoprotein J (clusterin) and Alzheimer's disease. Microsc. Res. Tech., 50: 305-315.

Collard, M.W. and Griswold, M.D. (1987) Biosynthesis and molecular cloning of sulfated glycoprotein 2 secreted by rat Sertoli cells. Biochemistry, 26(12): 3297-3303.

Conn, G., Bayne, M.L., Soderman, D.D., Kwok, P.W., Sullivan, K.A., Palisi, T.M., Hope, D.A. and Thomas, K.A. (1990) Amino acid and cDNA sequences of a vascular endothelial cell mitogen that is homologous to plateletderived growth factor. Proc. Natl. Acad. Sci. U.S.A., 87: $2628-2632$.

Danik, M., Champagne, D., Petit-Turcotte, C., Beffert, U. and Poirier, J. (1999) Brain lipoprotein metabolism and its relation to neurodegenerative disease. Crit. Rev. Neurobiol., 13(4): 357-407.

Dati, G., Quattrini, A., Bernasconi, L., Malaguti, M.C., Antonsson, B., Nicoletti, F., Alliod, C., Di Marco, R., Sagot, Y., Vitte, P.A., Hiver, A., Greco, B., Roach, A. and Zaratin, P.F. (2007) Beneficial effects of r-h-CLU on disease severity in different animal models of peripheral neuropathies. J. Neuroimmunol., 190: 8-17.

Dorle, D., Bewersdorf, J., Fruehwald-Schultes, B., Kern, W., Jelkmann, W., Born, J., Fehm, H.L. and Peters, A. (2002) Vascular endothelial growth factor: a novel endocrine defensive response to hypoglycemia. J. Clin. Endocrinol. Metab., 87: 835-840.

Duguid, J.R., Bohmont, C.W., Liu, N.G. and Tourtellotte, W.W. (1989) Changes in brain gene expression shared by scrapie and Alzheimer disease. Proc. Natl. Acad. Sci. U.S.A., 86(18): 7260-7264.

Endres, M., Laufs, U., Liao, J.K. and Moskowitz, M.A. (2004) Targeting eNOS for stroke protection. Trends Neurosci., 27: 283-289.

Ferrara, N. (2002) VEGF and the quest for tumour angiogenesis factors. Nat. Rev. Cancer, 2: 795-803.

Gerber, H.P., Mc Murtrey, A., Kowalski, J., Yan, M., Keyt, B.A., Dixit, V. and Ferrara, N. (1998) VEGF regulates endothelial cell survival by the $1 /$ KDR activation. J. Biol. Chem., 273: 30336-30343.

Góra-Kupilas, K. and Josko, J. (2005) The neuroprotective function of vascular endothelial growth factor (VEGF). Folia Neuropathol., 43(1): 31-39. 
Han, B.H., DeMattos, R.B., Dugan, L.L., Kim-Han, J.S., Brendza, R.P., Fryer, J.D., Kierson, M., Cirrito, J., Quick, K., Harmony, J.A.K., Aronow, B.J. and Holtzman, D.M. (2001) Clusterin contributes to caspase-3-independent brain injury following neonatal hypoxia-ischemia. Nat. Med., 7(3): 338-343.

Hashimoto, T., Zhang, X.M., Chen, B.Y. and Yang, X.J. (2006) VEGF activates divergent intracellular signaling components to regulte retinal progenitor cell proliferation and neuronal differentiation. Development, 133: 2201-2210.

Houck, K.A., Leung, D.W., Rowland, A.M., Winer, J. and Ferrara, N. (1992) Dual regulation of vascular endothelial growth factor bioavailability by genetic and proteolytic mechanisms. J. Biol. Chem., 267: 26031-26037.

Jenne, D.E. and Tschopp, J. (1989) Molecular structure and functional characterization of a human complement cytolysis inhibitor found in blood and seminal plasma: identity to sulfated glycoprotein 2, a constituent of rat testis fluid. Proc. Natl. Acad. Sci. U.S.A., 86(18): 7123-7127.

Jones, S.E. and Jomary, C. (2002) Clusterin. Int. J. Biochem. Cell Biol., 34: 427-431.

Józkowicz, A., Huk, I., Nigisch, A., Weigel, G., Dietrich, W., Motterlini, R. and Dulak, J. (2003) Heme oxygenase and angiogenic activity of endothelial cells: stimulation by carbon monoxide and inhibition by tin protoporphyrin-IX. Antioxid. Redox Signal., 5: 155-162.

Kyprianou, N., Martikainen, P., Davis, L., English, H.F. and Isaacs, J.T. (1991) Programmed cell death as a new target for prostatic cancer therapy. Cancer Surv., 11: 265-277.

Lakins, J., Bennett, S.A., Chen, J.H., Arnold, J.M., Morrissey, C., Wong, P., O'Sullivan, J. and Tenniswood, M. (1998) Clusterin biogenesis is altered during apoptosis in the regressing rat ventral prostate. J. Biol. Chem., 273(43): 27887-27895.

Lange, T., Guttmann-Raviv, N., Baruch, L., Machluf, M. and Neufeld, G. (2003) VEGF162, a new heparin-binding vascular endothelial growth factor splice form that is expressed in transformed human cells. J. Biol. Chem., 278: 17164-17169.

Lee, S., Jilani, S.M., Nikolova, G.V., Carpizo, D. and IruelaArispe, M.L. (2005) Processing of VEGF-A by matrix metalloproteinases regulates bioavailability and vascular patterning in tumors. J. Cell Biol., 169: 681-691.

Leskov, K.S., Klokov, D.Y., Li, J., Kinsella, T.J. and Boothman, D.A. (2003) Synthesis and functional analyses of nuclear clusterin, a cell death protein. J. Biol. Chem., 278(13): 11590-11600.

Leung, D.W., Cachianes, G., Kuang, W.J., Goeddel, D.V. and Ferrara, N. (1989) Vascular endothelial growth factor is a secreted angiogenic mitogen. Science, 246: 1306-1309.

Maines, M.D. (1997) The heme oxygenase system: a regulator of second messenger gases. Annu. Rev. Pharmacol. Toxicol., 37: $517-554$.

McLaughlin, L., Zhu, G., Mistry, M., Ley-Ebert, C., Stuart, W.D., Florio, C.J., Groen, P.A., Witt, S.A., Kimball, T.R., Witte, D.P., Harmony, J.A. and Aronow, B.J. (2000)
Apolipoprotein $\mathbf{J} /$ clusterin limits the severity of murine autoimmune myocarditis. J. Clin. Invest., 106(9): 1105-1113.

Morgan, T.E., Laping, N.J., Rozovsky, I., Oda, T., Hogan, T.H., Finch, C.E. and Pasinetti, G.M. (1995) Clusterin expression by astrocytes is influenced by transforming growth factor beta 1 and heterotypic cell interactions. J. Neuroimmunol., 58(1): 101-110.

Motterlini, R., Foresti, R., Bassi, R., Calabrese, V., Clark, J.E. and Green, C.J. (2000) Endothelial heme oxygenase-1 induction by hypoxia. Modulation by inducible nitric-oxide synthase and $S$-nitrosothiols. J. Biol. Chem., 275: 13613-13620.

Nakamura, E., Abreu-e-Lima, P., Awakura, Y., Inoue, T., Kamoto, T., Ogawa, O., Kotani, H., Manabe, T., Zhang, G.J., Kondo, K., Nosé, V. and Kaelin, W.G., Jr. (2006) Clusterin is a secreted marker for a hypoxia-inducible factorindependent function of the von Hippel-Lindau tumor suppressor protein. Am. J. Pathol., 168(2): 574-584.

Naskar, R. and Dreyer, E.B. (2001) New horizons in neuroprotection. Surv. Ophthalmol., 45(Suppl 3): S250-S255.

Nishijima, K., Ng, Y.S., Zhong, L., Bradley, J., Schubert, W., Jo, N., Akita, J., Samuelsson, S.J., Robinson, G.S., Adamis, A.P. and Shima, D.T. (2007) Vascular endothelial growth factor-A is a survival factor for retinal neurons and a critical neuroprotectant during the adaptive response to ischemic injury. Am. J. Pathol., 171(1): 53-67.

Oosthuyse, B., Moons, L., Storkebaum, E., Beck, H., Nuyens, D., Brusselmans, K., Van Dorpe, J., Hellings, P., Gorselink, M., Heymans, S., Theilmeier, G., Dewerchin, M., Laudenbach, V., Vermylen, P., Raat, H., Acker, T., Vleminckx, V., Van Den Bosch, L., Cashman, N., Fujisawa, H., Drost, M.R., Sciot, R., Bruyninckx, F., Hicklin, D.J., Ince, C., Gressens, P., Lupu, F., Plate, K.H., Robberecht, W., Herbert, J.M., Collen, D. and Carmeliet, P. (2001) Deletion of the hypoxia-response element in the vascular endothelial growth factor promoter causes motor neuron degeneration. Nat. Genet., 28: 131-138.

Orellana, D.I., Quintanilla, R.A., Gonzalez-Billault, C. and Maccioni, R.B. (2005) Role of the JAKs/STATs pathway in the intracellular calcium changes induced by interleukin- 6 in hippocampal neurons. Neurotox. Res., 8: 295-304.

Park, J.E., Keller, G.-A. and Ferrara, N. (1993) The vascular endothelial growth factor isoforms (VEGF): differential deposition into the subepithelial extracellular matrix and bioactivity of extracellular matrix-bound VEGF. Mol. Biol. Cell, 4: 1317-1326.

Pasinetti, G.M., Johnson, S.A., Oda, T., Rozovsky, I. and Finch, C.E. (1994) Clusterin (SGP-2): a multifunctional glycoprotein with regional expression in astrocytes and neurons of the adult rat brain. J. Comp. Neurol., 339(3): 387-400.

Pepper, M.S. (2001) Extracellular proteolysis and angiogenesis. Thromb. Haemost., 86: 346-355.

Pepper, M.S., Ferrara, N., Orci, L. and Montesano, R. (1992) Potent synergism between vascular endothelial growth factor and basic fibroblast growth factor in the induction of angiogenesis in vitro. Biochem. Biophys. Res. Commun., 189: $824-831$ 
Phillips, G.D., Stone, A.M., Jones, B.D., Schultz, J.C., Whitehead, R.A. and Knighton, D.R. (1994) Vascular endothelial growth factor (rhVEGF165) stimulates direct angiogenesis in the rabbit cornea. In Vivo, 8(6): 961-965.

Plouet, J., Schilling, J. and Gospodarowicz, D. (1989) Isolation and characterization of a newly identified endothelial cell mitogen produced by AtT20 cells. EMBO J., 8: 3801-3808.

Poltorak, Z., Cohen, T., Sivan, R., Kandelis, Y., Spira, G., Vlodavsky, I., Keshet, E. and Neufeld, G. (1997) VEGF145, a secreted vascular endothelial growth factor isoform that binds to extracellular matrix. J. Biol. Chem., 272: $7151-7158$.

Poon, S., Easterbrook-Smith, S.B., Rybchyn, M.S., Carver, J.A. and Wilson, M.R. (2000) Clusterin is an ATPindependent chaperone with very broad substrate specificity that stabilizes stressed proteins in a folding-competent state. Biochemistry, 39(51): 15953-15960.

Poon, S., Treweek, T.M., Wilson, M.R., Easterbrook-Smith, S.B. and Carver, J.A. (2002) Clusterin is an extracellular chaperone that specifically interacts with slowly aggregating proteins on their off-folding pathway. FEBS Lett., 513(2-3): 259-266.

Pucci, S., Bonanno, E., Pichiorri, F., Angeloni, C. and Spagnoli, L.G. (2004) Modulation of different clusterin isoforms in human colon tumorigenesis. Oncogene, 23(13): 2298-2304.

Raab, S. and Plate, K.H. (2007) Different networks, common growth factors: shared growth factors and receptors of the vascular and the nervous system. Acta Neuropathol., 113: 607-626.

Redondo, M., Villar, E., Torres-Muñoz, J., Tellez, T., Morell, M. and Petito, C.K. (2000) Overexpression of clusterin in human breast carcinoma. Am. J. Pathol., 157(2): 393-399.

Sawada, M., Sun, W., Hayes, P., Leskov, K., Boothman, D.A. and Matsuyama, S. (2003) Ku70 suppresses the apoptotic translocation of Bax to mitochondria. Nat. Cell Biol., 5(4): 320-329.

Schwarz, Q., Gu, C., Fujisawa, H., Sabelko, K., Gertsenstein, M., Nagy, A., Taniguchi, M., Kolodkin, A.L., Ginty, D.D., Shima, D.T. and Ruhrberg, C. (2004) Vascular endothelial growth factor controls neuronal migration and cooperates with Sema3A to pattern distinct compartments of the facial nerve. Genes Dev., 18: 2822-2834.

Senger, D.R., Galli, S.J., Dvorak, A.M., Perruzzi, C.A., Harvey, V.S. and Dvorak, H.F. (1983) Tumor cells secrete a vascular permeability factor that promotes accumulation of ascites fluid. Science, 219: 983-985.

Sone, H., Deo, B.K. and Kumagai, A.K. (2000) Enhancement of glucose transport by vascular endothelial growth factor in retinal endothelial cells. Invest. Ophthalmol. Vis. Sci., 41: 1876-1884.

Spitzer, M.S., Yoeruek, E., Sierra, A., Wallenfels-Thilo, B., Schraermeyer, U., Spitzer, B., Bartz-Schmidt, K.U. and Szurman, P. (2007) Comparative antiproliferative and cytotoxic profile of bevacizumab (Avastin), pegaptanib (Macugen) and ranibizumab (Lucentis) on different ocular cells. Graefes Arch. Clin. Exp. Ophthalmol., 245(12): 1837-1842.

Storkebaum, E., Lambrechts, D. and Carmeliet, P. (2004) VEGF: once regarded as a specific angiogenic factor, now implicated in neuroprotection. Bioessays, 26(9): 943-954.

Tezel, G. and Wax, M.B. (2004) Hypoxia-inducible factor lalpha in the glaucomatous retina and optic nerve head. Arch. Ophthalmol., 122: 1348-1356.

Tischer, E., Mitchell, R., Hartman, T., Silva, M., Gospodarowicz, D., Fiddes, J.C. and Abraham, J.A. (1991) The human gene for vascular endothelial growth factor. Multiple protein forms are encoded through alternative exon splicing. J. Biol. Chem., 266(18): 11947-11954.

Tolentino, M.J., Miller, J.W., Gragoudas, E.S., Chatzistefanou, K., Ferrara, N. and Adamis, A.P. (1996) Vascular endothelial growth factor is sufficient to produce iris neovascularization and neovascular glaucoma in a nonhuman primate. Arch. Ophthalmol., 114: 964-970.

Trougakos, I.P. and Gonos, E.S. (2002) Clusterin/apolipoprotein $\mathbf{J}$ in human aging and cancer. Int. J. Biochem. Cell Biol., 34: 1430-1448.

Van Lenten, B.J., Wagner, A.C., Navab, M. and Fogelman, A.M. (2001) Oxidized phospholipids induce changes in hepatic paraoxonase and ApoJ but not monocyte chemoattractant protein-1 via interleukin-6. J. Biol. Chem., 276(3): 1923-1929.

Vincenti, V., Cassano, C., Rocchi, M. and Persico, G. (1996) Assignment of the vascular endothelial growth factor gene to human chromosome 6p21.3. Circulation, 93: 1493-1495.

Wehrli, P., Charnay, Y., Vallet, P., Zhu, G., Harmony, J., Aronow, B., Tschopp, J., Bouras, C., Viard-Leveugle, I., French, L.E. and Giannakopoulos, P. (2001) Inhibition of post-ischemic brain injury by clusterin overexpression. Nat. Med., 7(9): 977-979.

Wiggins, A.K., Shena, P.J. and Gundlacha, A.L. (2003) Delayed, but prolonged increases in astrocytic clusterin (ApoJ) mRNA expression following acute cortical spreading depression in the rat: evidence for a role of clusterin in ischemic tolerance. Mol. Brain Res., 114: 20-30.

Wilson, M.R. and Easterbrook-Smith, S.B. (2000) Clusterin is a secreted mammalian chaperone. Trends Biochem. Sci., 25(3): 95-98.

Wong, P., Pineault, J., Lakins, J., Taillefer, D., Léger, J., Wang, C. and Tenniswood, M. (1993) Genomic organization and expression of the rat TRPM-2 (clusterin) gene, a gene implicated in apoptosis. J. Biol. Chem., 268(7): 5021-5031.

Yamashita, T., Sawamoto, K., Suzuki, S., Suzuki, N., Adachi, K., Kawase, T., Mihara, M., Ohsugi, Y., Abe, K. and Okano, H. (2005) Blockade of interleukin-6 signaling aggravates ischemic cerebral damage in mice: possible involvement of Stat 3 activation in the protection of neurons. J. Neurochem., 94: 459-468.

Zenkel, M., Kruse, F.E., Jünemann, A.G., Naumann, G.O. and Schlötzer-Schrehardt, U. (2006) Clusterin deficiency in eyes with pseudoexfoliation syndrome may be implicated in the aggregation and deposition of pseudoexfoliative material. Invest. Ophthalmol. Vis. Sci., 47(5): 1982-1990. 